\title{
Salmonella typhimurium translocates flagellin across intestinal epithelia, inducing a proinflammatory response
}

\author{
Andrew T. Gewirtz, ${ }^{1}$ Peter O. Simon, Jr., ${ }^{1}$ Clare K. Schmitt, ${ }^{2}$ Laura J. Taylor, ${ }^{3}$ \\ Curt H. Hagedorn, ${ }^{3}$ Alison D. O’Brien, ${ }^{2}$ Andrew S. Neish, ${ }^{1}$ and James L. Madara ${ }^{1}$
}

1Epithelial Pathobiology Division, Department of Pathology, Emory University School of Medicine, Atlanta, Georgia, USA
${ }^{2}$ Department of Microbiology and Immunology, Uniformed Services University of the Health Sciences, Bethesda, Maryland, USA
${ }^{3}$ Digestive Diseases Division, Department of Medicine, Emory University School of Medicine, Atlanta, Georgia, USA

Address correspondence to: Andrew T. Gewirtz, Department of Pathology, Emory University, Atlanta, Georgia 30322, USA. Phone: (404) 712-9885; Fax: (404) 727-8538; E-mail: agewirt@emory.edu.

Received for publication June 5, 2000, and accepted in revised form November 13, 2000.

This study investigated whether soluble paracrine factors mediated Salmonella-induced IL-8 expression in polarized model intestinal epithelia. We found that the basolateral media of model epithelia that had been apically infected with Salmonella typhimurium for a short period (10 minutes) could activate IL-8 secretion in virgin model epithelia, demonstrating that a proinflammatory factor (PIF) was indeed present. Initial characterization found that PIF was a heat-stable protein with a molecular mass of about $50 \mathrm{kDa}$ that acts on the basolateral, but not apical, surface of model intestinal epithelia to elicit IL-8 secretion. PIF was not present in the media of model epithelia stimulated with other inducers of IL-8 secretion (TNF- $\alpha$ or carbachol) but was present in S. typhimurium supernatants, indicating PIF is of bacterial origin. PIF was purified from bacterial culture supernatants by anion/cation exchange chromatography and SDS-PAGE and found by using microsequencing to be the protein flagellin. In support of this finding, flagellin-deficient S. typhimurium mutants did not secrete detectable levels of PIF (i.e., a bioactivity that induced IL-8 secretion when placed basolaterally on model epithelia). Furthermore, viable flagellin-deficient mutant organisms $(f l i C / f l j B$ and $f l b D)$ failed to elicit IL-8 secretion when added apically to model intestinal epithelia. These findings indicate that translocation of flagellin across epithelia, subsequent to apical epithelial-S. typhimurium interaction, is likely a major means of activating a mucosal inflammatory response.

J. Clin. Invest. 107:99-109 (2001).

\section{Introduction}

Infection of humans by the enteric pathogen Salmonella typhimurium generally results in severe abdominal cramping and diarrhea. These symptoms may largely result from the mucosal immune response elicited by this pathogen. Specifically, colonization of the human intestine by $S$. typhimurium leads to infiltration of polymorphonuclear leukocytes (PMNs) into the intestinal epithelium culminating in the formation of an intestinal crypt abscess (1). The action of these PMNs on the epithelium and the loss of barrier function that results are thought to be key events in mediating the clinical manifestations of Salmonellosis (for review see ref. 2). Interestingly, the pathobiology of the acute flares of inflammatory bowel disease appears to be very similar to that of Salmonellosis (2), though efforts to identify a responsible organism (or other provoking agonist) have not been successful.

We have shown previously that the first cells colonized by $S$. typhimurium, the intestinal epithelial cells, play an important role in directing the PMN movement that occurs in response to this pathogen. Specifically, in response to contact between S. typhimurium and their apical membranes, intestinal epithelial cells synthesize and secrete a panel of chemokines that participate in the recruitment of immune cells (3-5). One of the best characterized of these chemokines is the neutrophil chemoattractant IL-8. IL-8 is secreted basolaterally by polarized model intestinal epithelia such that it imprints a haptotactic gradient for PMNs on the subepithelial matrix, thus likely directing the movement of extravasated PMNs through the lamina propria (6).

We have shown that $S$. typhimurium induces IL-8 secretion via $\mathrm{Ca}^{++}$-mediated activation of the transcription factor NF- $\kappa B$ (7). However, the molecular interactions that activate these epithelial signaling pathways remain undefined. Morphologic studies of S. typhimurium adherence to intestinal epithelia indicate that this pathogen does not bind uniformly to epithelial cells, but rather a subset of epithelial cells bound (or internalized) many bacteria whereas most epithelial cells appeared free of attached (or internalized) organisms (8). However, the level of IL-8 secreted by S. typhimuri$u m$-infected model epithelia is similar to that observed in response to soluble agonists such as TNF- $\alpha$ that ought to uniformly activate these epithelial cells (7), suggesting that noncolonized epithelial cells might be involved in the IL-8 secretory response. Therefore, we 
speculated that S. typhimurium might induce the epithelial secretion of a proinflammatory factor (PIF) that would induce NF-KB activation and IL-8 secretion from cells without associated bacteria. The data herein show that, indeed, the basolaterally conditioned media of apically infected model intestinal epithelia possess the ability to elicit IL-8 secretion from unstimulated epithelia. However, to our surprise, we found the responsible factor was not of epithelial origin but rather related to translocation of the bacterial protein flagellin across epithelia where it subsequently acts on the basolateral surface of these cells to induce proinflammatory signaling events.

\section{Methods}

Bacterial culture. S. typhimurium $\mathrm{X}_{3306}$ and SL3201 (wildtype strains), S. typhimurium $\mathrm{PhoP}^{c}$ and Hils (invasiondefective strains), and Escherichia coli F-18 (normal gut strain) were maintained as described previously $(4,6,9)$. Flagellar mutants were prepared from the SL3201 strain (10). The $f l b D$ gene (flagella master operon) was mutated by P22 transduction of flhD:TN10 from strain KK2040 (kind gift of K. Kutsukake, Hiroshima University, Hiroshima, Japan) (11). The flagellar mutants SL3201 fljB:MudJ,SL3201 fliC:Tn10, and SL3201 fljB:MudJ fliC:Tn10 were described previously (12). SL3201 $f l i C / f l j B$ was transformed with the $f l i C$-containing plasmid pKK1012 (13) and the $f l j B$-containing plasmid pKK1025I (14), both of which were received from K. Kutsukake. S. typhimurium mutants invA and invG, and their parent SR11, were received from Katherine Reed (Institute for Animal Health, Berkshire, United Kingdom). These studies also used seven strains of "normal" gut E. coli. E. coli F-18 (serotype rough:K1:H5) (15) was received from Beth McCormick (Harvard University Medical School, Boston, Massachusetts, USA). E. coli HS was previously isolated from human feces (16). Additionally, five gut $E$. coli isolates obtained from the feces of healthy donors, as described previously (17), at the University of Illinois Medical Center (Chicago, Illinois, USA) were generously supplied by Gail Hecht (these five strains were assigned numbers $4-8$ by Hecht). Cultures were prepared for experimental use under nonagitated, microaerophilic conditions by culturing $0.01 \mathrm{ml}$ of stationary-phase culture in $10 \mathrm{ml}$ of Luria broth (LB) overnight ( $15-18$ hours) at $37^{\circ} \mathrm{C}$, as outlined previously $(4,18)$. Bacterial overnight cultures were concentrated 33-fold in HBSS, with 10 mM HEPES, pH 7.4.

Cell culture. Model intestinal epithelia were prepared using T84 or HT29-Cl.19A cells as described previously (19) on $0.33 \mathrm{~cm}^{2}$ permeable supports. Model epithelia were used 6-14 days after plating after verification (for T84) that they had achieved a transepithelial electrical resistance of at least $1000 \Omega \mathrm{cm}^{2}$.

Isolation of PIF (from basolaterally conditioned media of model intestinal epithelia). T84 model intestinal epithelia were washed three times in HBSS and placed into wells containing $0.3 \mathrm{ml}$ HBSS. HBSS $(0.1 \mathrm{ml})$ was added to the apical reservoir, and cells were allowed to equilibrate for $30-45$ minutes at $37^{\circ} \mathrm{C}$. After equilibration, epithelia were transferred to empty wells, apical buffer discarded, and $25 \mu \mathrm{l}$ of a freshly prepared S. typhimurium suspension was added apically for 5 minutes. Epithelia were then transferred to wells containing $0.3 \mathrm{ml}$ HBSS and further incubated at $37^{\circ} \mathrm{C}$ for 55 minutes (unless otherwise specified). The model epithelia were then discarded and the basolateral media collected. The basolateral, PIF-containing media was subsequently filtered (0.22- $\mu \mathrm{m}$ pore; Millipore Corp., Bedford, Massachusetts, USA), boiled at $100^{\circ} \mathrm{C}$ for 20 minutes (except in initial experiments), then stored for later use at $-20^{\circ} \mathrm{C}$.

Isolation of crude PIF (from bacterial culture supernatants). Bacterial overnight cultures were washed two times and concentrated 33 -fold in HBSS. Samples were incubated for 1 hour at $37^{\circ} \mathrm{C}$. After the incubation, the bacteria were removed by centrifugation, and the PIF-containing supernatant was filtered $(0.22 \mu \mathrm{m}$-pore; Millipore Corp.) and boiled at $100^{\circ} \mathrm{C}$ for 20 minutes. The PIF-containing supernatant was then stored for later use at $-20^{\circ} \mathrm{C}$.

Bacterial-induced IL-8 secretion. IL-8 secretion in response to live apically added bacteria was assayed as described previously $(7,20)$.

PIF-, TNF- $\alpha$-, and carbachol-induced IL-8 secretion. IL-8 was induced in the same manner except that PIF (various specified dilutions), recombinant TNF- $\alpha$ (R\&D Systems Inc., Minneapolis, Minnesota, USA), and carbachol $(100 \mu \mathrm{M})$, were added basolaterally. Epithelia were then incubated for 5 hours at $37^{\circ} \mathrm{C}$. IL-8 levels were determined by ELISA, as described previously (9).

Transepithelial electrical resistance measurements. Apical and basolateral reservoirs of T-84 intestinal epithelia were connected to a voltage clamp using a pair of calomel and $\mathrm{Ag}-\mathrm{AgCl}$ electrodes via agar bridges as described previously (19). The changes in potential differences generated by the passage of $25-\mu \mathrm{A}$ current pulses were measured, and resistance was calculated as outlined previously (19). Clostridium difficile toxin A (a generous gift of Richard J. Obiso, Jr., East Tennessee State University, Johnson City, Tennessee, USA) (40 $\mathrm{ng} / \mathrm{ml}$ ) and PIF were added basolaterally.

Purification of PIF: production of PIF-containing culture supernatants. Three liters of LB was inoculated with 3 $\mathrm{ml}$ of a stationary-phase bacterial culture and incubated overnight (15-18 hours) at $37^{\circ} \mathrm{C}$. The bacterial cultures were then concentrated 100 -fold by centrifugation in HBSS and incubated for 1 hour at $37^{\circ} \mathrm{C}$. After the incubation, the bacteria were removed by centrifugation, the PIF-containing supernatant was filtered (0.22- $\mu \mathrm{m}$ pore; Millipore Corp.) and boiled at $100^{\circ} \mathrm{C}$ for 20 minutes. The PIF-containing supernatant was then stored for later use at $-20^{\circ} \mathrm{C}$.

Purification of PIF: gel filtration chromatography. PIF was purified using a Biologic HR chromatography system (Bio-Rad Laboratories, Hercules, California, USA). This system was connected to a flow adapter (Bio-Rad Laboratories) and glass Econo-column $(75 \mathrm{~cm}, 124 \mathrm{ml}$; BioRad Laboratories) packed with $125 \mathrm{ml}$ of Superose- 6 
resin (Amersham Pharmacia Biotech, Piscataway, New Jersey, USA). The column was equilibrated with HBSS, and $0.5 \mathrm{ml}$ of sample was run at $0.1 \mathrm{ml} / \mathrm{min}$. One-milliliter fractions were collected. Bio-Rad Laboratories' gel filtration standards were used for calibration.

Purification of PIF: cation-exchange extraction and anionexchange chromatography. PIF containing supernatants $(30 \mathrm{ml})$ were concentrated to approximately $10 \mathrm{ml}$ using Amicon (Millipore Corp.) 30-kDa filters. The $\mathrm{pH}$ of the PIF-containing solution was adjusted to $\mathrm{pH}$ 6.0 and incubated (shaking) for 30 minutes at room temperature with $0.5 \mathrm{ml}$ of S-Sepharose ( $\mathrm{pH}$ 6.0; Amersham Pharmacia Biotech). After the incubation, the S-Sepharose resin was pelleted by centrifugation. The PIF-containing supernatant was then diluted fivefold in $20 \mathrm{mM}$ Tris- $\mathrm{HCl}, \mathrm{pH} 8.0$, and applied to a 2-ml Q-Sepharose column (pH 8.0) (Bio-Rad Laboratories). The column was washed with $15 \mathrm{ml}$ of $20 \mathrm{mM}$ Tris- $\mathrm{HCl}(\mathrm{pH} \mathrm{8.0)}$ at a rate of $2 \mathrm{ml} / \mathrm{min}$. A $100 \mathrm{ml}$, 0.0-1.0 M NaCl gradient was applied to the loaded column at a flow rate of $2 \mathrm{ml} / \mathrm{min}$. Fractions $(1 \mathrm{ml})$ were collected and analyzed for activity by IL-8 induction in T-84 model intestinal epithelia.

Surface flagellin was isolated by mechanical shearing with an Omni homogenizer (21). S. typhimurium and $E$. coli (serotypes 026:B6 and 055:B5) LPS were obtained from Sigma Chemical Co. (St. Louis, Missouri, USA). E. coli F-18 LPS was purified by microextraction using proteinase $\mathrm{K}$ digestion as described previously (22). These LPS preparations were verified to be bioactive by measuring their ability to induce IL-8 secretion in human umbilical vein endothelial cells (HUVECs).

SDS-PAGE. SDS-PAGE was performed with precast 4-20\% Tris- $\mathrm{HCl}$ polyacrylamide gels (Bio-Rad Laboratories). Western blotting of flagellin was performed using mAb 15D8 (IGEN International Inc., Gaithersburg, Maryland, USA) to E. coli flagellin that crossreacts with $S$. typhimurium flagellin. Briefly, proteins from SDS-PAGE gels were transferred to nitrocellulose, blocked overnight, and blotted with anti-flagellin $(1 \mu \mathrm{g} / \mathrm{ml})$ followed by goat anti-mouse antibody $(1 \mu \mathrm{g} / \mathrm{ml}$; Amersham Pharmacia Biotech). Detection was achieved by enhanced chemiluminescence.

Data processing. Error bars (where shown) are the means plus or minus SEM of an experiment performed in triplicate (i.e., using three model epithelia per condition) and are representative of three separate experiments.

\section{Results}

S. typhimurium-epithelial interactions result in the release of a proinflammatory protein. S. typhimurium contact with the apical surface of model intestinal epithelia induces an increase in the epithelial intracellular $\left[\mathrm{Ca}^{++}\right]$that leads to activation of the transcription factor NF- $\mathrm{KB}$ and subsequently synthesis and secretion of IL-8 (7). The latter (i.e., NF- $\kappa \mathrm{B}$ activation, IL-8 secretion), but not proximal $\mathrm{Ca}^{++}-$ mediated portion of this signaling pathway, is shared by the proinflammatory cytokine TNF- $\alpha$. This led to the hypothesis that the $\left[\mathrm{Ca}^{++}\right]$increase induced by $S$. typhimurium could cause the epithelial exocytosis of a proinflammatory mediator that could then activate IL-8 expression by a mechanism similar (or identical) to that used by TNF- $\alpha$. Thus, we collected the conditioned media of S. typhimurium-infected model epithelia 1 hour after application of the bacteria and examined the ability of these samples to activate IL- 8 secretion in virgin (i.e., noninfected) epithelia. After filtration through a $0.2-\mu \mathrm{m}$ pore filter to remove bacteria, the apically conditioned media was transferred to the apical surface of virgin epithelia while the basolaterally conditioned media was transferred to the basolateral aspect of an additional virgin epithelia. Five hours later, we measured the IL-8 concentration in the basolateral reservoir because IL-8 secretion is known to a occur in a basolaterally polarized manner regardless of the type or location of stimulus (23). As shown in Figure 1a, transfer of basolaterally conditioned media, but not apically conditioned media, significantly induced IL-8 secretion, indicating the existence of a PIF. Nonphysiological transfer of apically conditioned media to the basolateral surface of virgin epithelia, but not transfer of basolaterally conditioned media to the apical surface, also induced IL-8 secretion (data not shown), suggesting the response to PIF, rather than the release of PIF, was polarized (addressed later in this article). PIF bioactivity (i.e., ability to induce IL-8 secretion) became detectable in the basolateral media 15 minutes after apical addition of the
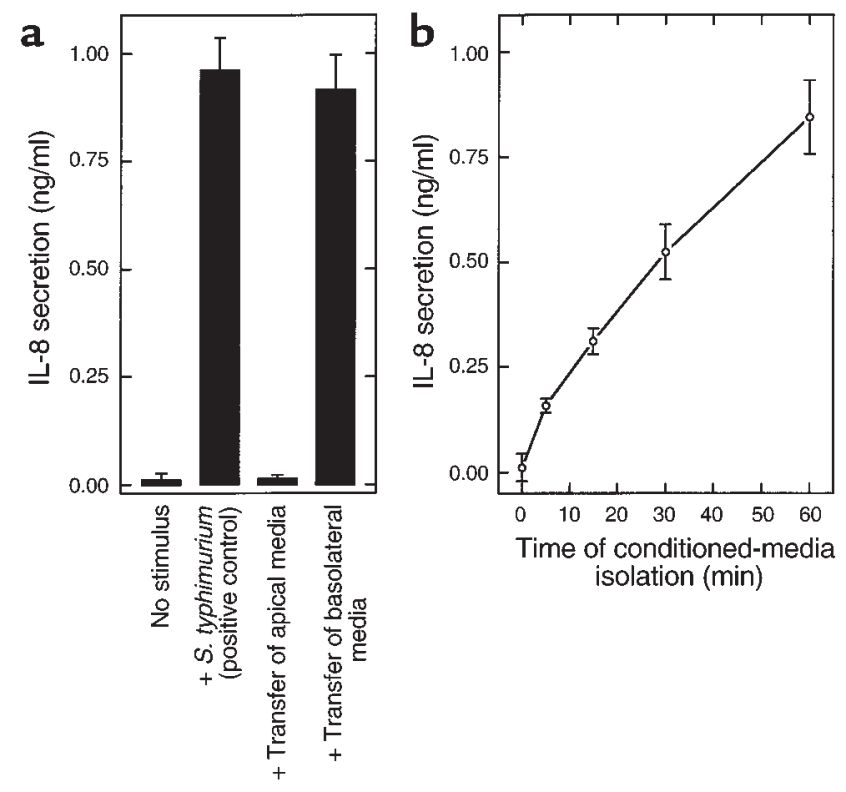

\section{Figure 1}

Basolaterally conditioned media of S. typhimurium-infected epithelia induce IL-8 secretion in virgin epithelia. Model intestinal epithelia were apically exposed to $S$. typhimurium. The epithelial media was then transferred to untreated model epithelia. IL- 8 was assayed in basolateral media 5 hours later. (a) Media was transferred maintaining its polarity of origin (i.e., apical to apical, basolateral to basolateral) 1 hour after addition of bacteria. Live S. typhimurium (added apically for 5 hours) served as a positive control. (b) Basolateral media was transferred at the indicated time after addition of bacteria. 
a
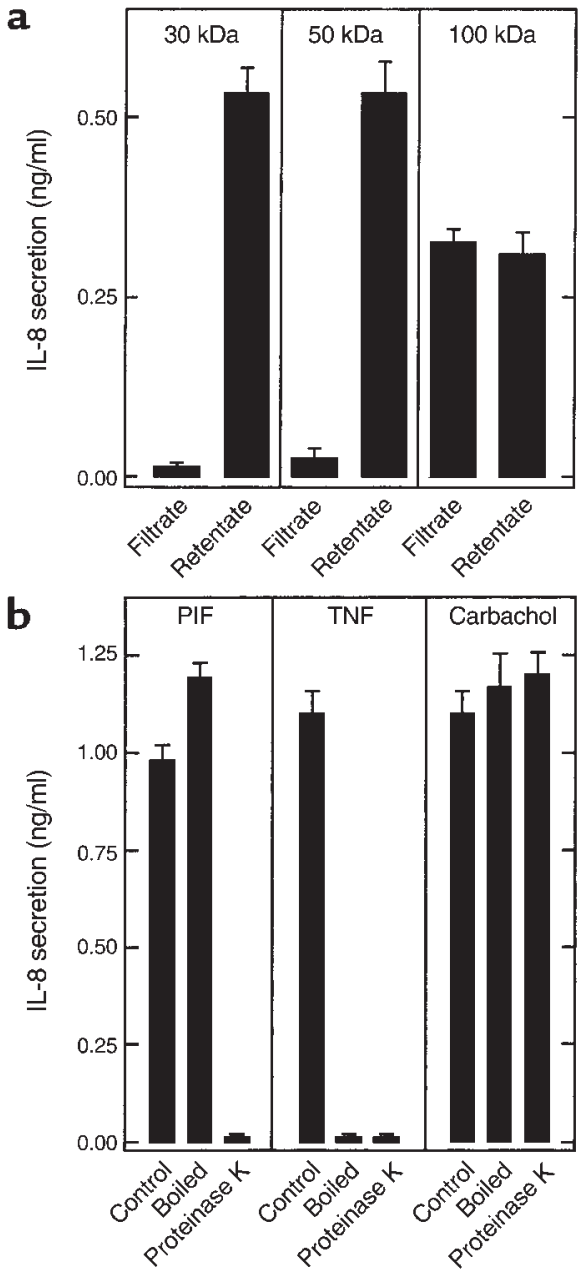

Figure 2

$\mathrm{PIF}$ is a heat-stable protein. Samples from $\mathbf{a}$ and $\mathbf{b}$ were applied basolaterally to model epithelia, and their ability to induce IL-8 secretion over a 5-hour period was measured. (a) PIF was centrifuged through indicated Amicon concentrators followed by dilution of retentate to original volume. (b) PIF, TNF- $\alpha(20 \mathrm{ng} / \mathrm{ml})$, and carbachol $(100 \mu \mathrm{M})$ were subjected to boiling (20 minutes) or proteinase $\mathrm{K}$ treatment $(1 \mathrm{mg} / \mathrm{ml}$ for 1 hour followed by 10 minutes of boiling to inactivate proteinase $K)$.

bacteria, and the amount of PIF bioactivity increased thereafter (Figure 1b). Induction of IL-8 secretion was henceforth used as a measure of PIF bioactivity.

We next began a biochemical characterization of PIF. To obtain an approximate size estimation of PIF, we filtered it using molecular weight (MW) cut-off filters. PIF activity was completely retained in the retentate of 50 $\mathrm{kDa}$ (and lower) filters and variably retained by higher MW cut-off filters (Figure 2a). Next, we measured PIF sensitivity to 20 minutes of boiling. This treatment did not reduce the ability of PIF to induce IL-8 secretion in model epithelia (i.e., PIF bioactivity) (Figure 2b). In contrast, heating TNF- $\alpha$ in this manner resulted in a complete loss of its bioactivity. We next measured the effect of treating PIF with proteinase K. PIF bioactivity was completely destroyed by this treatment (Figure 2b), indicating that PIF contains an essential protein com- ponent. To ensure that the proteinase $\mathrm{K}$ was not interfering with the IL-8 induction assay (even though the proteinase $\mathrm{K}$ was inactivated by boiling), we verified that this proteinase had no effect on IL-8 induction by carbachol. Lastly, we verified that PIF behavior regarding MW cut-off filters did not differ if PIF was first boiled before being applied to these filters (data not shown), suggesting that PIF was indeed a fairly large molecule rather than a smaller molecule being bound by a larger one. Together, these results indicate that PIF is a heatstable protein with an approximate MW of 50-100 kDa.

PIF release is not induced by nonbacterial agonists. To investigate whether PIF was of epithelial, rather than bacterial, origin we attempted (unsuccessfully) to induce epithelial PIF release with nonbacterial agonists. Basolaterally conditioned media of TNF- $\alpha$-treated model epithelia were isolated 45 minutes after addition of this agonist. These samples were then boiled to inactivate the TNF- $\alpha$ without harming any PIF that may have been secreted. Samples isolated in this manner did not display any IL-8-inducing bioactivity (Figure 3), indicating that TNF- $\alpha$ does not induce the release of PIF. Next, we investigated whether PIF release could be induced by the acetylcholine receptor agonist carbachol in accordance with our hypothesis that PIF is secreted by $\mathrm{Ca}^{++}$-mediated exocytosis (both carbachol and $S$. typhimurium induce $\mathrm{Ca}^{++}$mobilization). Since basolaterally conditioned media of carbachol-stimulated epithelia contains carbachol, which, like PIF, is heat stable, such samples were applied to epithelia with and without treatment with proteinase $\mathrm{K}$. Basolaterally conditioned media of carbachol-treated epithelia did not exhibit any proteinase $\mathrm{K}$-sensitive induction of IL8 secretion in virgin epithelia (Figure 3 ). To be certain that the residual carbachol had not simply masked a PIF response, this carbachol was removed (90\%) by filtration through a $10-\mathrm{kDa} \mathrm{MW}$ cut-off filter. The retentate exhibited no more ability to induce IL-8 secretion than a similarly treated retentate (carbachol-containing) that had not been preconditioned by epithelia, indicating that carbachol, like TNF- $\alpha$, does not induce PIF secretion from model epithelia.

PIF bioactivity can be isolated from bacterial supernatants. Having failed to obtain PIF secretion in a bacteria-free assay, we next sought to identify a PIF bioactivity in supernatants of bacteria that had not interacted with epithelial cells. The same S. typhimurium inoculum that we typically use to induce epithelial IL-8 secretion was harvested by centrifugation, washed in HBSS, and incubated for 1 hour at $37^{\circ} \mathrm{C}$. The culture was then centrifuged and the supernatant filtered through a $0.2-\mu \mathrm{M}$ filter to remove residual bacteria. This sample potently induced IL-8 secretion when applied to the basolateral surface of model intestinal epithelia (Figure 4a). Like PIF isolated from bacterial-epithelial interactions, the proinflammatory bioactivity derived from bacterial supernatant retained the ability to induce epithelial IL-8 expression when boiled, was sensitive to proteinase $\mathrm{K}$, and was retained by $30 \mathrm{kDa}$ cut- 


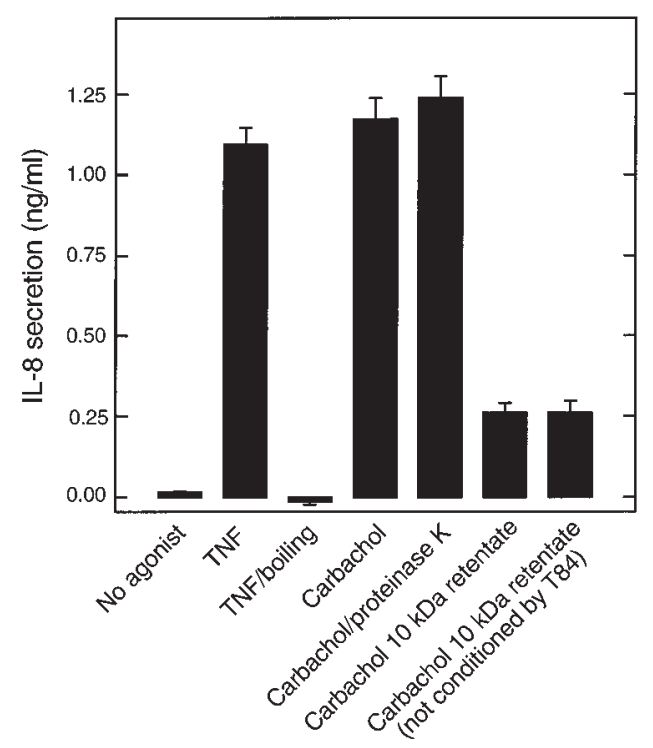

Figure 3

Epithelia do not release PIF in response to TNF- $\alpha$ or carbachol. Model epithelia were basolaterally treated with TNF- $\alpha(20 \mathrm{ng} / \mathrm{ml})$ or carbachol $(100 \mu \mathrm{M})$. One hour later, basolateral media (which still contained TNF- $\alpha$ or carbachol) was isolated and subjected to boiling or proteinase $\mathrm{K}$ treatment where indicated (as described in Figure 2). Additionally, carbachol-containing media (and an equimolar solution of carbachol that had not been exposed to epithelia) were concentrated tenfold over a $10-\mathrm{kDa}$ Amicon concentrator and then rediluted to the original volume. Samples were then basolaterally exposed to fresh model epithelia, and their ability to induce IL-8 secretion over a 5 -hour period was measured.

off filters (Figure $4 \mathrm{~b}$ ). These results indicated that PIF is likely of bacterial origin. We next investigated whether bacterial LPS was involved in this response by using polymyxin B-coated agarose to remove this compound from PIF (24). Removal of endotoxin in this manner did not result in any loss of PIF activity (control and polymyxin B bead-treated PIF induced $1.54 \pm 0.23$ and $1.64 \pm 0.27 \mathrm{ng} / \mathrm{ml}$ IL-8 secretion, respectively). To be sure that the polymyxin $\mathrm{B}$ agarose did actually remove endotoxin, we verified that our treatment abolished (99.98 $\pm 3.2 \%$ inhibition) the abil- ity of a solution of LPS to activate IL-8 secretion in HUVECs (a well-characterized LPS responsive cell type). Thus, LPS is not a component of PIF.

PIF represents flagellin. We first sought to obtain PIF's MW by using gel-filtration chromatography. Bacterial supernatant was filtered $(0.2-\mu \mathrm{m}$ pore size), applied to a Superose column (13- $\mu \mathrm{m}$ particle size), and fractions assayed for PIF activity by measuring their ability to induce IL-8 secretion from model epithelia. PIF bioactivity eluted as a single peak with a molecular mass of about $50 \mathrm{kDa}$. We purified PIF by an ion exchange chromatography, using as a starting material bacterial supernatants that had been boiled, filtered, and concentrated tenfold with a 30-kDa MW cut-off filter. By performing test bindings, we observed that PIF bioactivity readily bound to anion exchange, but not cation exchange resins. Thus, we used cation exchange beads to remove proteins not related to PIF bioactivity and then applied the resulting sample to a Q-Sepharose anion-exchange column. PIF bioactivity eluted from the anion-exchange column as a single peak that upon SDS-PAGE analysis contained a prominent $50-\mathrm{kDa}$ band (Figure 5). This band was digested with trypsin, extracted from the gel, analyzed using amino acid sequencing (Edman degradation), and the sequences FNSAITNLG and TTSYT obtained. A search of the Salmonella database indicated that the first sequence was from FliC and the second from FljB. Thus, the band contained both of the two known S. typhimurium isoforms of the protein flagellin, the primary structural component of flagella.

We next investigated whether we had indeed purified the protein responsible for PIF bioactivity. S. typhimuri$u m$ has two highly homologous genes for flagellin, and growth conditions may dictate which isoform is expressed. Functional flagella can be made from either protein. We thus generated S. typhimurium strains deficient in flagellin (lacking the genes for both $\mathrm{fliC}$ and/or $f l B B$. We isolated supernatant from these strains and their wild-type parent and measured the ability of these supernatants to induce IL-8 secretion when applied to the basolateral surface of model intestinal epithelia. In
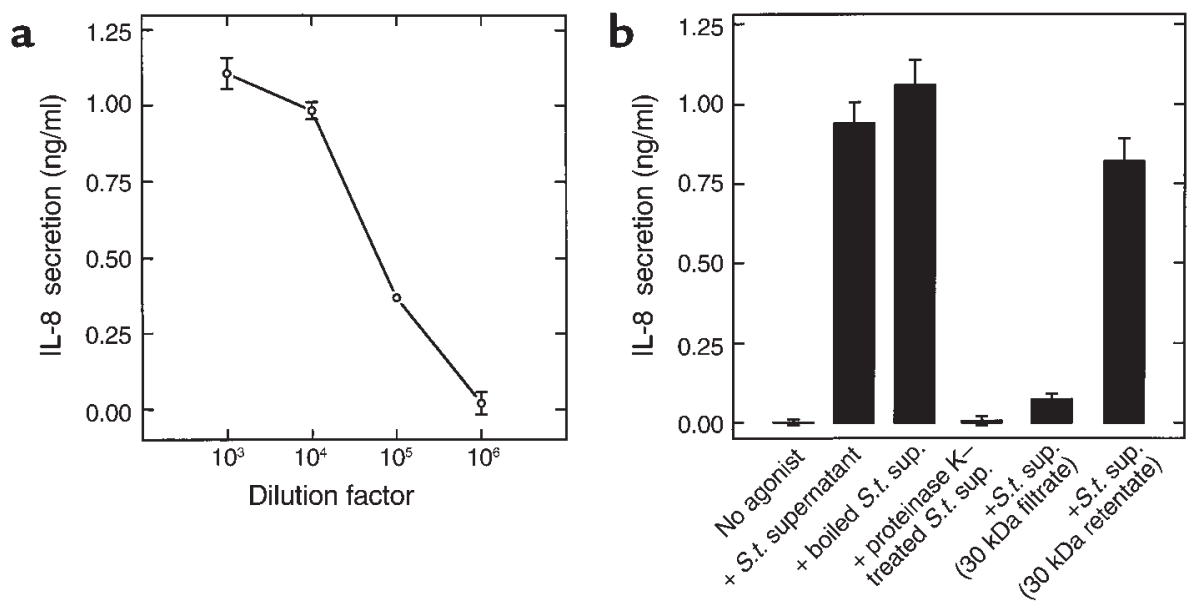

Figure 4

Bacteria release a PIF with the same properties as that isolated from bacterial-epithelial interactions. An overnight culture of S. typhimurium (S.t.) was pelleted, washed two times by centrifugation, and resuspended in HBSS $\left(10^{10} \mathrm{CFU} / \mathrm{ml}\right)$. One hour later, bacterial supernatant was isolated and basolaterally applied to model epithelia where its ability to induce IL-8 secretion was measured.(a) Supernatant was applied over a range of concentrations. (b) Bacterial supernatant was subjected to indicated treatments (as described in Figure 2) before being applied to epithelia.

The Journal of Clinical Investigation | January 2001 | Volume 107 | Number 1 


\section{Figure 5}

Purification of PIF revealed it to be the protein flagellin. (a) Outline of PIF purification method. Numbers in parentheses correspond to lane numbers in b. (b) Coomassie-stained gel showing PIF at various stages of purification. Lane 1: MW markers (in kDa: 205, 120, 84, 52, 36, 30, 22, 7). Lane 2: S. typhimurium supernatant (i.e., starting material; too dilute to detect any bands). Lane 3: Sample concentrated tenfold over $30-\mathrm{kDa}$ cut-off Amicon filter. Lane 4: Concentrated sample after incubation with S-Sepharose cation exchange beads. Lane 5: The peak bioactive anion exchange fraction. This band was sequenced and found to be flagellin. FT, flow through.
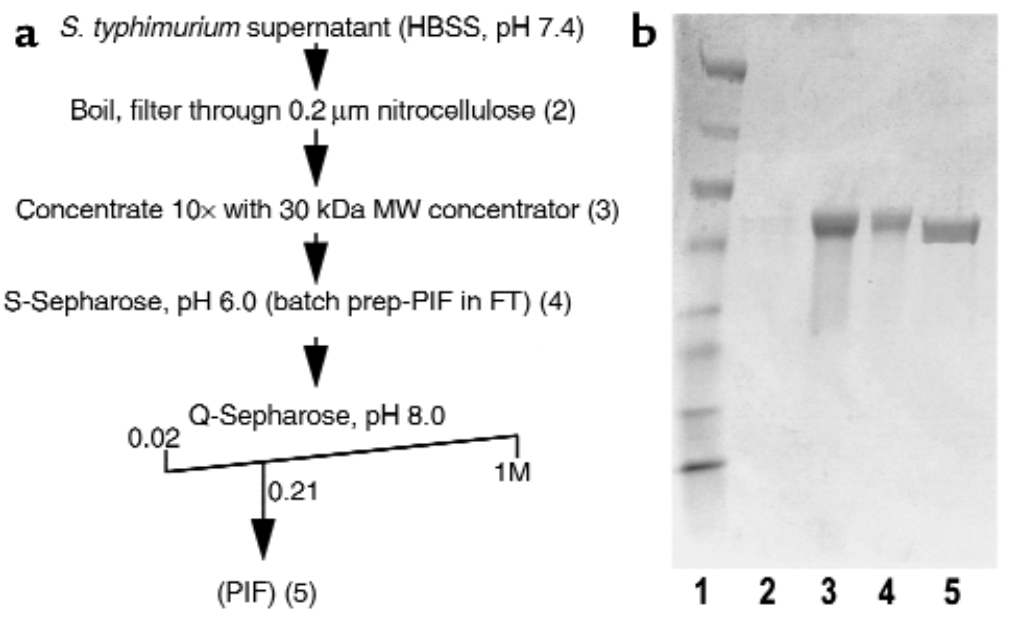

striking contrast to the wild-type strain, the flagellindeficient mutant $(f l i C / f l j B)$ supernatant did not display any IL-8-inducing bioactivity, even when added at a 1,000 -fold greater concentration (Figure 6). We also analyzed whether S. typhimurium deficient in either, but not both, $f l i C$ or $f l j B$ secreted the IL-8-inducing factor. The supernatants of either of these two strains contained significant, albeit somewhat reduced, levels of IL-8-inducing activity when compared with the wild-type strain. Thus, although our wild-type cultures (originating from a single colony) contain a mix of FliC and $\mathrm{FljB}$ (perhaps the result of a growth-phase change during overnight incubation), either flagellin isoform can function as a PIF. The relatively reduced potencies of supernatants lacking FliC or FljB probably resulted from a lower concentration of flagellin rather than synergy between the two flagellin isoforms present in the wild-type supernatant (see below). Lastly, we verified that we could rescue the inability of flagellin-deficient $S$. typhimurium supernatants to induce IL-8 secretion by transforming them with plasmids encoding $f l i C$ or $f l j B$. As shown in Figure $7 \mathrm{~b}$, transformation with either flagellin gene restored the ability of the organism's supernatants to induce IL-8 secretion, further confirming our conclusion that $\mathrm{FliC}$ and $\mathrm{Flj}$ B are proinflammatory factors.

Bacterial-induced PIF responses require epithelial translocation of flagellin. Many flagellated bacteria will release flagellin into their media, and thus supernatants of most Salmonella strains would be expected to activate IL-8 secretion when applied (basolaterally, see below) to epithelia. Indeed, as shown in Figure 7, supernatants from every Salmonella strain we tested (except flagellar mutants), and five out of seven strains of nonpathogenic gut $E$. coli, contained flagellin as indicated by Western blotting (Figure 7a) and induced epithelial IL-8 secretion (Figure 7b) when applied basolaterally. Diluting some of these bacterial supernatants and then measuring their ability to induce IL-8 secretion indicated that the supernatants of these flagellated bacterial strains contained similar levels of PIF/flagellin bioactivity (Figure 7c). Furthermore, the PIFs isolated from these strains had the same biochemical properties as PIF/flagellin isolated from wild-type $S$. typhimurium (resistance to boiling, sensitivity to proteinase K; data not shown), indicating that flagellin was likely responsible for this IL-8 induction.

However, in contrast to their supernatants, the PhoPc mutant and the E. coli strains themselves elicited very little IL-8 secretion when added apically (i.e., physiologically) to model epithelia (refs. 9, 17 and Figure 7d). We reasoned that this apparent contradiction could perhaps be explained by epithelia exhibiting a polarized response to flagellin; i.e., perhaps epithelia could respond only to flagellin that reaches its basolateral surface. This was indeed the case because flagellin added apically to model epithelia failed to elicit IL-8 secretion even when added at 100-fold greater concentration than was necessary to elicit a significant response when added basolaterally (Figure 8). Therefore, as we normally add bacteria only to the apical (lumenal) surface (consistent with the physiology of the intestine), there would only be a response to flagellin if and when it is

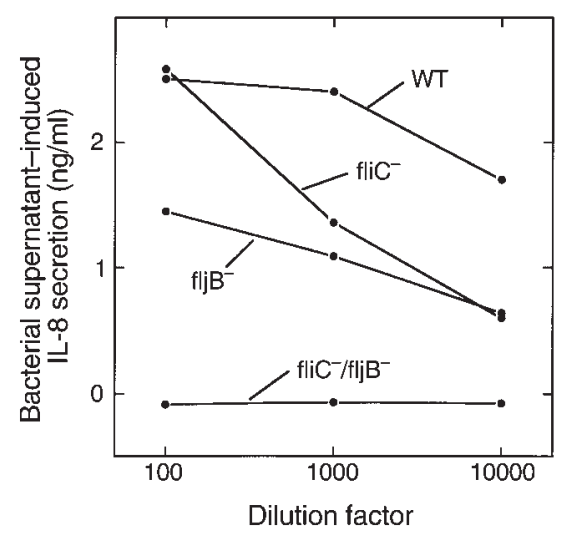

Figure 6

Flagellin-deficient S. typhimurium do not release an IL-8 inducing bioactivity. Bacterial supernatants were isolated (as described in Figure 4) from wild-type (WT) S. typhimurium or mutant strains lacking $\mathrm{fliC}$ and/or fljB ("-" indicates the gene that is missing). Supernatants were then diluted as indicated, applied basolaterally to model epithelia, and IL- 8 secretion assayed 5 hours later. 


\section{a}
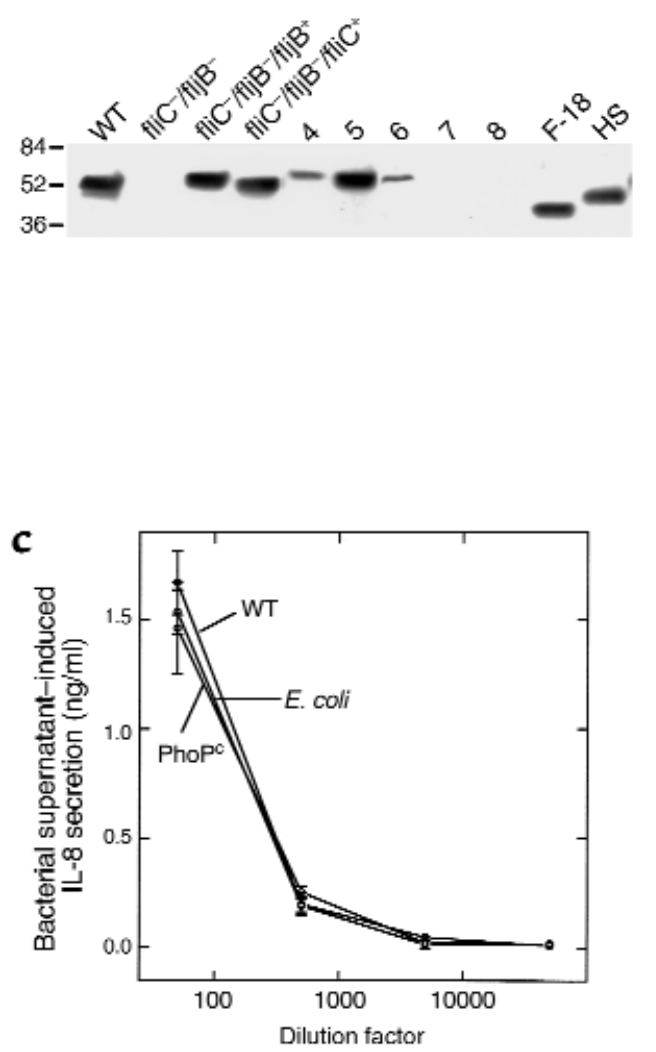
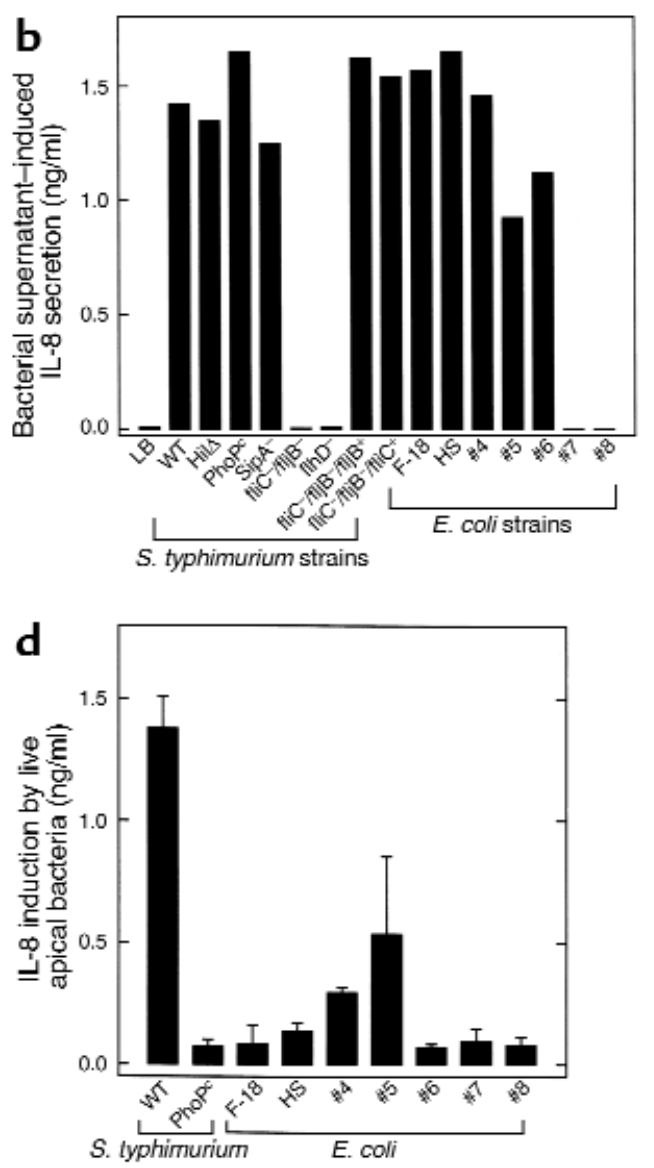

Figure 7

Various bacteria release PIF/flagellin. An overnight culture of the indicated bacterial strain was pelleted, washed two times by centrifugation, and resuspended in $\mathrm{HBSS}\left(10^{10} \mathrm{CFU} / \mathrm{ml}\right)$. One hour later, bacterial supernatant was isolated. Flagellin-deficient strain (flic-/ $\left.\mathrm{fljB}^{-}\right)$ transformed with plasmids encoding $\mathrm{fliC}$ and $\mathrm{flj} B$ is indicated by $\mathrm{fliC}^{-} / \mathrm{fljB}^{-} / \mathrm{fliC}^{+}$and $\mathrm{fliC}^{-} / \mathrm{fljB}^{-} / \mathrm{fljB}^{+}$, respectively. (a) Supernatants were analyzed by SDS-page/immunoblotting using a mAb to flagellin. (b-d) Model epithelia were placed in HBSS. (b) Bacterial supernatants were applied basolaterally at a 1:100 dilution to model epithelia. IL-8 secretion was assayed 5 hours later. (c) Bacterial supernatants were applied basolaterally, at indicated dilution, to model epithelia. IL-8 secretion was assayed 5 hours later. (d) Live bacteria were applied to apical reservoir ( $10^{8}$ bacteria/ epithelia). IL-8 secretion was assayed 5 hours later.

translocated across the epithelia. However, the fact that a flagellin-mediated bioactivity was isolated from the basolateral supernatants (Figure 1) indicates that indeed such translocation does occur. Additionally, Western blotting of such basolateral-conditioned media indicate that these samples do indeed contain detectable levels of flagellin within 30 minutes after apical addition of the bacteria (Figure 9a).

We next sought to gain some insight into how flagellin translocation might occur. Flagellin itself, added apically in the absence of bacteria, was unable to translocate across model epithelia (Figure 9b). Nor could flagellin translocation be mediated by a flagellin-secreting strain of normal gut $E$. coli. Furthermore, using our more sensitive and quantitative indicator of flagellin translocation (i.e., detecting flagellin by its IL-8-inducing bioactivity), we found that flagellin-deficient $S$. typhimurium could not translocate added soluble flagellin (IL-8 induction by basolateral media of epithelia apically col-

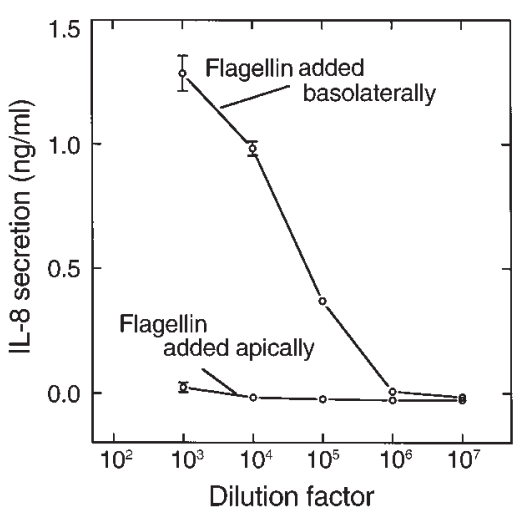

Figure 8

Basolateral flagellin, but not apical flagellin, induces IL-8 secretion. Flagellin was isolated from $S$. typhimurium supernatant as described in Methods, diluted as indicated, and added to the indicated reservoir of model epithelia, and IL-8 secretion was measured 5 hours later. 
a

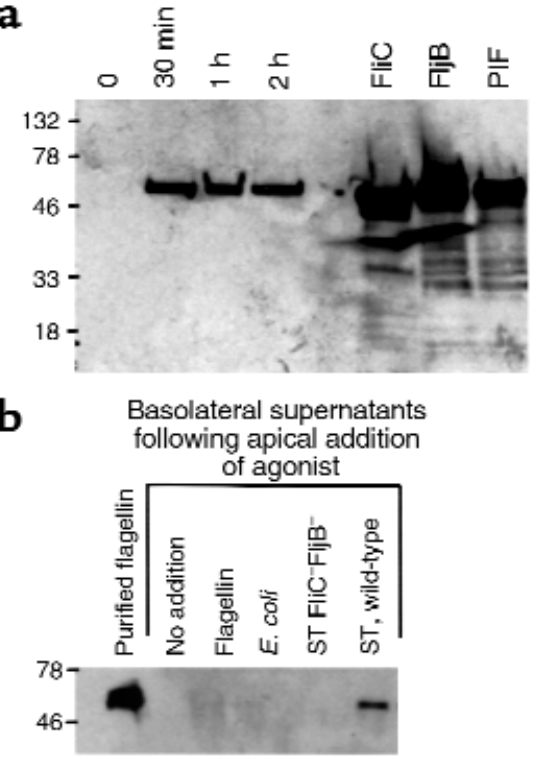

\section{Figure 9}

Flagellin rapidly appears in the basolateral supernatants of apically infected model epithelia. Model intestinal epithelia were apically exposed to indicated bacteria. Basolateral epithelial supernatants were isolated and Western blotted for flagellin. (a) Supernatants were isolated at the indicated time after addition of S. typhimurium. Isolated surface flagellin from S. typhimurium expressing FliC or FljB and PIF were directly Western blotted (i.e., not exposed to epithelia) to serve as positive controls. (b) Supernatants were isolated 1 hour after exposure to indicated organism or purified flagellin $(100 \mathrm{ng} / \mathrm{ml})$.

onized with $\mathrm{fliC}^{-} / \mathrm{fljB}^{-}$S. typhimurium in the presence of apical soluble flagellin was $7.3 \pm 1.5 \%$ of wild-type values). These results suggest that flagellin translocation is mediated by $S$. typhimurium interacting directly with flagellin and intestinal epithelia.

To begin to characterize the microbial determinants that might be required for flagellin translocation, the ability of previously characterized invasion-defective $S$. typhimurium mutants to mediate flagellin translocation was measured. Two regulatory mutants, $\mathrm{PhoPc}^{\mathrm{c}}$ (constitutive activation of the $\mathrm{PhoP} / \mathrm{PhoQ}$ regulatory system) (25) and Hil $\Delta$ (spontaneous 8-kb deletion including prgH) (26), as well as the mutants invA and inv $G$, both lacking structural components of the type III secretory apparatus (27), were used. We have shown previously that the Hils, but not $\mathrm{PhoP}^{c}$, mutant retains the ability to induce IL-8 secretion $(7,9)$. Concomitantly, Hils, but not $\mathrm{PhoP}^{\mathrm{c}}$, could mediate the translocation of flagellin as measured by biodetection (Figure 10). Nonpathogenic E. coli F-18 was also unable to translocate biodetectable levels of flagellin. The S. typhimurium mutants invA and inv $G$ exhibited somewhat diminished, but not abolished, ability to translocate flagellin when compared with the wildtype strain (Figure 10). When assayed in parallel for invasion, invA and $i n v G$ were found to be internalized approximately $80 \%$ and $70 \%$, respectively, less than a wild-type strain (internalization for wild-type parent, $i n v A$, and $i n v G$ was $30.7 \pm 6.5,6.0 \pm 1$, and $9.3 \pm 4 \times 10^{4}$ bacteria per $0.33 \mathrm{~cm}^{2}$ epithelia). Together, these results indicate that the type III secretory-mediated interactions between $S$. typhimurium and epithelia may modulate, but are not absolutely required for, flagellin translocation. Moreover, whereas many flagellated Gram-negative organisms secrete flagellin, this flagellin will only activate an inflammatory response when an organism can translocate this protein across the epithelia. Thus, the ability to translocate flagellin may be an important determinant in whether a bacteria will elicit an inflammatory response.

Primary S. typhimurium induction of IL-8 occurs by a flagellin-mediated mechanism. We next sought to better assess the biological relevance of flagellin induction of epithelial IL-8 secretion. First, we measured the concentration dependence of this response. Flagellin purified from wild-type S. typhimurium (likely a mix of FliC and $\mathrm{FljB}$ ) induced detectable IL-8 secretion at concentrations below $1 \mathrm{ng} / \mathrm{ml}$ and had an $\mathrm{ID}_{50}$ of about 2 $\mathrm{ng} / \mathrm{ml}$ (Figure 11a). FljB (flagellin purified from fliC $S$. typhimurium) had an $\mathrm{ID}_{50}$ of $1.5 \mathrm{ng} / \mathrm{ml}$, whereas FliC (purified from the $f l j B$ strain) had an $\mathrm{ID}_{50}$ of $2.4 \mathrm{ng} / \mathrm{ml}$. This is more potent than IL-8 induction elicited by an equal concentration of the potent proinflammatory agonist TNF- $\alpha$. In contrast, these cells do not secrete detectable levels of IL-8 in response to basolaterally added S. typhimurium LPS (no response was observed at any concentration we tested, the highest being 500 $\mu \mathrm{g} / \mathrm{ml})$, analogous to the results of nonpolarized epithelia $(28,29)$ or polarized epithelia treated apically $(4,17)$. Similarly, we did not observe detectable IL-8

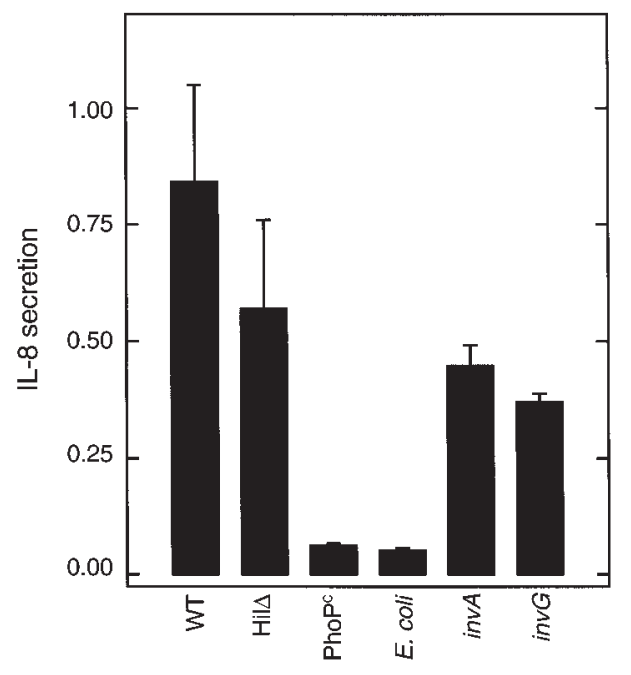

Figure 10

Flagellin translocation by S. typhimurium mutants. Model epithelia were apically exposed to wild-type (WT) S. typhimurium or indicated mutant or nonpathogenic E. coli. for 45 minutes. After exposure to bacteria, basolateral supernatants were collected, boiled, filtered (through $2-\mu \mathrm{M}$ pore size filter), and transferred to basolateral surface of virgin epithelia. IL-8 secretion was measured 5 hours later using ELISA. Wild-type represents $P$ hoPc parent (14028s). An indistinguishable result was obtained using invA parent SR-11 (not shown). 
secretion in response to two commercially available $E$. coli LPS preparations or LPS that we isolated from $E$. coli F-18. Thus, whereas LPS is thought to be important for activating subepithelial (i.e., lamina propria) macrophages, flagellin may play an important role in activating epithelial orchestration of an inflammatory response when the epithelial barrier has been breached. We next assessed whether flagellin would stimulate IL-8 production from model epithelia made from cell lines other than T84. HT29cl.19A model epithelia also secreted IL-8 in response to flagellin (unstimulated and $100 \mathrm{ng} / \mathrm{ml}$ flagellin-treated HT29cl19A epithelia secreted $0.045 \pm .25$ and $1.53 \pm 0.45 \mathrm{ng} / \mathrm{ml} \mathrm{IL-8,} \mathrm{respec-}$ tively), indicating this response was not unique to a single cell line. Like TNF- $\alpha$, and unlike bacterial toxins such as $C$. difficile toxin A, flagellin does not itself cause significant changes in epithelial barrier function within the time it induces IL-8 induction (Figure 11b), indicating it is likely activating an inflammatory response rather than simply being cytotoxic.

Lastly, we investigated what portion of the IL-8 secretory response induced by $S$. typhimurium, applied in a physiologically polarized manner (i.e., apically), was accounted for by flagellin. Specifically, we examined the ability of the $f l i C / f l j B$ mutant, as well as an $f l h D$ mutant (flagellar master operon), to induce IL-8 secretion when applied apically to model epithelia. Similar to their supernatants (Figure 6 and 7), neither of these live organisms elicited significant levels of IL-8 secretion (Figure 11c) from model epithelia. In contrast, strains lacking only FliC or FljB elicited IL-8 secretion to an extent that was only marginally reduced from the wild-type parent strain. Furthermore, transformation of the flagellin-deficient strain with plasmids that encode either $f l i C$ or $f l j B$ fully restored the bacteria's ability to induce this response. Thus, flagellin is not merely an amplifier of IL-8 expression. Rather, flagellin appears to play a major role in activating epithelial orchestration of the immune inflammatory response.

\section{Discussion}

This report shows that the protein flagellin, the primary structural component of E. coli and Salmonella flagella (30), is a potent inducer of IL-8 expression in model intestinal epithelia when this protein obtains access to the basolateral (serosal) aspect of such epithelia. While it is not entirely clear why bacteria release soluble flagellin (it is unlikely to be shedding from flagella that depolymerize because gentamicin-killed bacteria do not release significant amounts of soluble flagellin; data not shown), it appears to occur among a variety of bacterial strains. Expression of flagellin by the pathogen S. typhimurium was found to be essential for this organism to induce the epithelial secretion of IL-8. As IL-8 expression is both a marker and mediator of mucosal inflammation in the intestine (31-33), this finding has profound implications on the understanding of the mechanisms by which enteric organisms can activate mucosal inflammation.

The mucosal surface of the human colon is very heavily colonized with Gram-negative organisms, many of which (e.g., nonpathogenic E. coli) likely release flagellin. Thus, flagellin is normally likely to be ubiquitous at the lumenal epithelial membrane domain. In contrast, since the intestinal epithelium is normally impenetrable to most bacteria, the basolateral membrane environment (i.e., lamina propria) likely is normally a flagellin-free environment. In this context, our
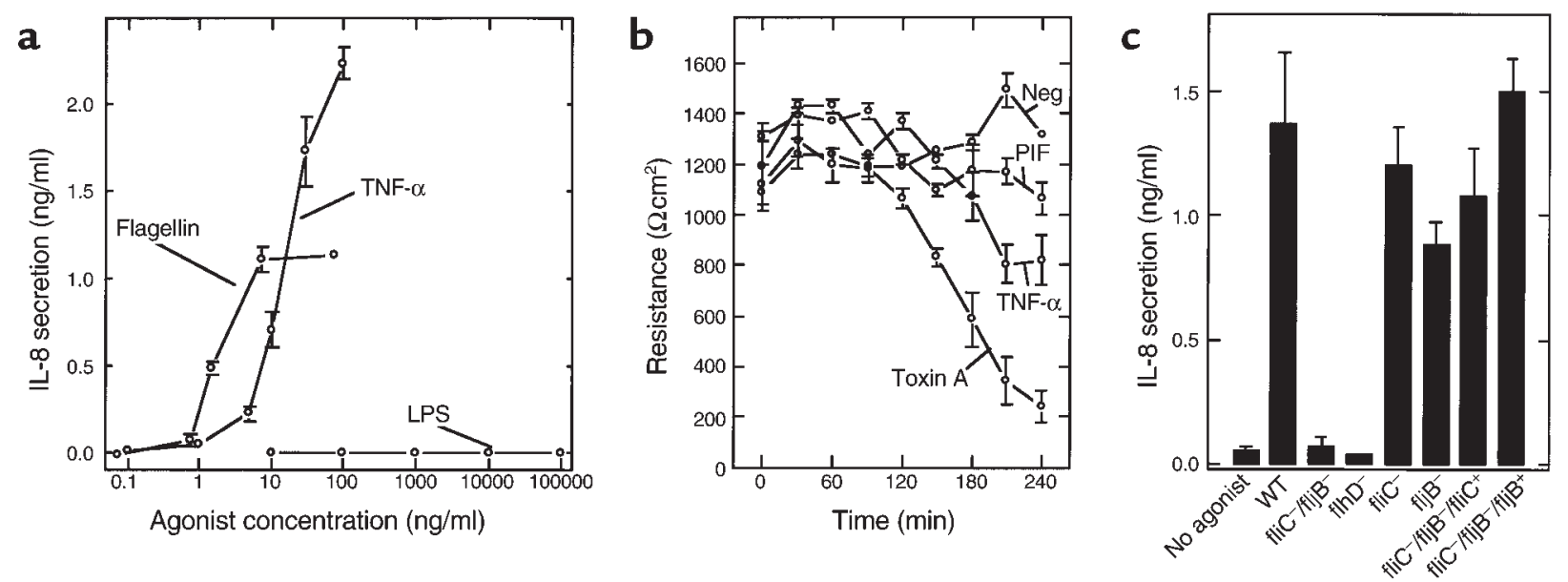

\section{Figure 11}

Flagellin is a potent inducer of IL-8 secretion, does not drop transepithelial electrical resistance (TER), and is essential for IL-8 secretion induced by S. typhimurium. (a) Model epithelia were basolaterally exposed to indicated concentration of LPS, flagellin, or TNF- $\alpha$. IL-8 secretion was assayed 5 hours later. (b) Model epithelia were exposed (basolaterally) to HBSS (Neg), PIF (1:40 of sample used in Figure 4), TNF$\alpha(20 \mathrm{ng} / \mathrm{ml})$, or C. difficile toxin A (Toxin A). TER was measured as described in Methods. (c) Model epithelia were apically colonized for 45 minutes with live indicated wild-type (WT) S. typhimurium or indicated mutant strain. Nonadherent bacteria were removed by washing three times with HBSS. IL-8 secretion was measured 5 hours after initial exposure to bacteria. Flagellin-deficient $\left.\operatorname{strain}_{(\mathrm{fliC}}^{-} / \mathrm{fljB}^{-}\right)$transformed with plasmids encoding $\mathrm{fliC}$ and $\mathrm{fljB}$ is indicated by $\mathrm{fliC}^{-} / \mathrm{fljB}^{-} / \mathrm{fliC}^{+}$and $\mathrm{fliC}^{-} / \mathrm{fljB}^{-} / \mathrm{fljB}^{+}$, respectively. 
observation that flagellin exposed to the basolateral, but not apical (lumenal), surface of model epithelia induces IL-8 secretion has significance for two reasons. First, the pathogen S. typhimurium was able to mediate the translocation of flagellin across the epithelium, and such translocated flagellin was able to activate proinflammatory gene expression in uninfected epithelial cells. That flagellin-deficient $S$. typhimurium did not elicit detectable IL-8 secretion suggests that this mechanism of epithelial activation may be essential for epithelial orchestration of a mucosal inflammatory response. While flagellin-deficient $S$. typhimurium also exhibit considerably reduced ability to invade epithelial cells (34) (in our model system invasion of $\mathrm{fliC} / \mathrm{flj} B$ was $4.3 \pm 1.1 \times 10^{3}$ bacteria/epithelia compared to 26.0 $\pm 3.1 \times 10^{3}$ bacteria for the parent strain), this fact is insufficient to explain their inability to induce IL-8 secretion, because we have shown these events can be uncoupled (9). Second, in intestinal diseases associated with epithelial dysfunction such as inflammatory bowel disease (IBD), there is diminished barrier function that may allow the lumenal contents access to the serosal surface of the epithelium. This could result in flagellin inducing epithelial cells to orchestrate an inflammatory response in the absence of any normally pathogenic organisms. This proposed role for flagellin in IBD is similar to that proposed for LPS acting on lamina propria macrophages. While monocytic cells recently have also been shown to secrete cytokines in response to flagellin (35), the epithelial response to flagellin may be of particular importance since epithelial cells are, by far, the most numerous cells in the intestinal mucosa, and, in contrast to macrophages, are relatively unresponsive to LPS $(17,28,29)$, even when added basolaterally (our results herein).

Currently, the mechanism by which flagellin translocation occurs is unknown, but some insights can be drawn. Neither flagellin isolated from S. typhimurium or E. coli were, by themselves, able to cross intact model epithelium. Rather, only live S. typhimurium were able to mediate the translocation of this protein, indicating direct bacterial-epithelial interaction is involved. It seems unlikely that $S$. typhimurium causes this rather large molecule to pass through epithelial cell tight junctions because the transepithelial electrical resistance (a direct indicator of passive permeability) drops only modestly in response to this invasive pathogen (4). It is possible that S. typhimurium that invade epithelia may simply basolaterally exit the epithelium and secrete flagellin at such a location. However, analogous to the case for IL-8 induction, invasion may not be required for flagellin translocation because we have found that the invasion-defective Hil $\Delta$ mutant was able to induce PIF/flagellin translocation (refs. 9 and 29 and herein). Thus, it is possible that S. typhimurium attached to the apical membrane can translocate flagellin into epithelial cells and that this protein could be trafficked to exit the basolateral membrane. S. typhimurium is well-known to secrete effector molecules into epithelial cells, partic- ularly through a type III secretion apparatus. S. typhimurium, lacking essential structural components of this apparatus, displayed diminished flagellin translocation, suggesting a modulating role for this structure. Flagellin is normally secreted from S. typhimurium through a secretory apparatus highly homologous to the type III secretory apparatus that this pathogen uses to inject other molecules into host cells (36), allowing us to envisage the possibility that the flagellin could be exported into epithelial cells via this apparatus. We are currently engaged in developing strategies and techniques to investigate such possible mechanisms.

If flagellin is indeed playing a role in inflammation associated with IBD, understanding the mechanism by which this molecule activates such proinflammatory pathways may allow the development of novel therapeutic strategies for treating this disorder. While flagellin exists as polymerized flagellar filaments when embedded in bacteria, the fact that bacterial supernatants were filtered through a $0.2-\mu \mathrm{M}$ pore-size filter and that we obtained a nondenatured MW using gel filtration chromatography of about $50 \mathrm{kDa}$ suggests that the proinflammatory activity described herein is coming from flagellin monomers rather than filaments. While it is possible that the basolateral epithelial membrane may somehow be more permeable to flagellin than its apical counterpart, it seems more likely that there is a "flagellin receptor" expressed on the basolateral, but not apical, membrane of intestinal epithelial cells. Supporting this idea, we have observed that flagellin induces a $\left[\mathrm{Ca}^{++}\right]$increase in model epithelia in response to basolateral flagellin (data not shown). Thus, whereas we had speculated that epithelial $\mathrm{Ca}^{++}$might play a role in releasing a PIF, rather $\mathrm{Ca}^{++}$may play a role in the epithelial response to the PIF flagellin, likely by leading to activation of the transcription factor NF- $\mathrm{KB}$ similar to the signals we have shown that are activated by S. typhimurium (7). The existence of such a "flagellin receptor" awaits confirmation and identification.

In conclusion, our inquiry into how S. typhimurium interaction with a relatively small number of epithelial cells is able to mediate a robust proinflammatory response indicates that the bacterial protein flagellin serves as an important soluble mediator. There did not seem to be much difference in the ability of flagellin from different Salmonella (or even E. coli) strains to induce this response, but rather only those strains that could mediate the translocation of this protein had proinflammatory bioactivity. It seems reasonable to envisage that epithelial cells have evolved a response to flagellin at its basolateral surface as a means of the host realizing its primary barrier has been breached and that a mucosal immune inflammatory response is warranted. Such a system would be effective in sensing the presence of a wide variety of bacteria, regardless of how the organisms translocated across the epithelium. However, it is also possible that S. typhimurium may have evolved an invasion-independent mechanism to translocate flagellin, thus activating chemokine 
expression as a mucosal inflammatory response eventuating in diarrhea and may offer a disseminating advantage to this organism. Regardless, normal gut microflora flagellin may, through its actions on the intestinal epithelium, play a role in inflammation associated with IBD and hence may serve as a strategic target for treating this disorder.

\section{Acknowledgments}

Invaluable advice on purifying PIF was received from Jan Pohl and Randy Mrsny. Purified C. difficile toxin A was provided Richard J. Obiso, Jr. We thank Lauren Collier for helpful discussion of this work. This work was supported by NIH grants DK-9800 (A. Gewirtz), DK-2792 (A. Gewirtz), AI33525 (A. O’Brien), CA63640 (C. Hagedorn), HL60033 (A. Neish), and DK-4762 (J. Madara).

1. Takeuchi, A. 1967. Electron microscope studies of experimental Salmonella infection. Am. J. Pathol. 50:109-119.

2. Madara, J.L. 1997. Review article: pathobiology of neutrophil interactions with intestinal epithelia. Aliment. Pharmacol. Ther. 11(Suppl. 3):57-62.

3. McCormick, B.A., Parkos, C.A., Colgan, S.P., Carnes, D.K., and Madara, J.L. 1998. Apical secretion of a pathogen-elicited epithelial chemoattractant (PEEC) activity in response to surface colonization of intestinal epithelia by Salmonella typhimurium. J. Immunol. 160:455-456.

4. McCormick, B.A., Colgan, S.P., Archer, C.D., Miller, S.I., and Madara, J.L. 1993. Salmonella typhimurium attachment to human intestinal epithelial monolayers: transcellular signalling to subepithelial neutrophils. $J$. Cell Biol. 123:895-907.

5. Jung, H.C., et al. 1995. A distinct array of proinflammatory cytokines is expressed in human colon epithelial cells in response to bacterial invasion. J. Clin. Invest. 95:55-65.

6. McCormick, B., et al. 1995. Surface attachment of Salmonella typhimurium to intestinal epithelia imprints the subepithelial matrix with gradients chemotactic for neutrophils. J. Cell Biol. 131:1599-1608.

7. Gewirtz, A.T., et al. 2000. Salmonella typhimurium induces epithelial IL-8 expression via $\mathrm{Ca}^{2+}$-mediated activation of the NF- $\kappa \mathrm{B}$ pathway. J. Clin. Invest. 105:79-92.

8. Giannasca, K.T., Giannasca, P.J., and Neutra, M.R. 1996. Adherence of Salmonella typhimurium to Caco-2 cells: identification of a glycoconjugate receptor. Infect. Immun. 64:135-145

9. Gewirtz, A.T., Siber, A.M., Madara, J.L., and McCormick, B.A. 1999. Orchestration of neutrophil movement by intestinal epithelial cells in response to salmonella typhimurium can be uncoupled from bacterial internalization. Infect. Immun. 67:608-617.

10. Hoiseth, S.K., and Stocker, B.A. 1981. Aromatic-dependent Salmonella typhimurium are non-virulent and effective as live vaccines. Nature. 291:238-239.

11. Kutsukake, K., and Iino, T. 1994. Role of the FliA-FlgM regulatory system on the transcriptional control of the flagellar regulon and flagellar formation in Salmonella typhimurium. J. Bacteriol. 176:3598-3605.

12. Schmitt, C.K., Darnell, S.C., and O'Brien, A.D. 1996. The attenuated phenotype of a Salmonella typhimurium flgM mutant is related to expression of FliC flagellin. J. Bacteriol. 178:2911-2915.

13. Inoue, Y.H., Kutsukake, K., Iino, T., and Yamaguchi, S. 1989. Sequence analysis of operator mutants of the phase- 1 flagellin-encoding gene, fliC, in Salmonella typhimurium. Gene. 85:221-226.

14. Kutsukake, K., Nakao, T., and Iino, T. 1985. A gene for DNA invertase and an invertible DNA in Escherichia coli K-12. Gene. 34:343-350.
15. McCormick, B.A., Franklin, D.P., Laux, D.C., and Cohen, P.S. 1989. Type 1 pili are not necessary for colonization of the streptomycin-treated mouse intestine by type 1 piliated Escherichia coli F-18 and E. coli K-12. Infect. Immun. 57:3022-3029.

16. DuPont, H.L., et al. 1971. Pathogenesis of Escherichia coli diarrhea. N. Engl.J. Med. 285:1-9.

17. Savkovic, S.D., Koutsouris, A., and Hecht, G. 1997. Activation of NF-kap$\mathrm{paB}$ in intestinal epithelial cells by enteropathogenic Escherichia coli. Am. J. Physiol. 273:C1160-C1167.

18. Lee, C.A., and Falkow, S. 1990. The ability of Salmonella to enter mammalian cells is affected by bacterial growth state. Proc. Natl. Acad. Sci. USA. 87:4304-4308.

19. Madara, J.L., Colgan, S.P., Nusrat, A., Delp, C., and Parkos, C.A. 1992. A simple approach to measurement of electrical parameters of cultured epithelial monolayers: use in assessing neutrophil epithelial interactions. Journal of Tissue Culture Methods. 15:209-216.

20. Gewirtz, A.T., et al. 1998. Pathogen-induced chemokine secretion from model intestinal epithelium is inhibited by lipoxin A4 analogs. J. Clin. Invest. 101:1860-1869.

21. Lockman, H.A., and Curtiss, R.D. 1990. Salmonella typhimurium mutants lacking flagella or motility remain virulent in $\mathrm{BALB} / \mathrm{c}$ mice. Infect. Immun. 58:137-143.

22. Apicella, M.A., Griffiss, J.M., Schneider, H. 1997. Isolation and characterization of lipopolysaccharides, lipooligosaccharides, and lipid A. In Bacterial pathogenesis. V.L. Clark and P.M. Bavoil, editors. Academic Press. San Diego, California, USA. 123-133.

23. Eckmann, L., et al. 1993. Differential cytokine expression by human intestinal epithelial cell lines: regulated expression of interleukin-8. Gastroenterology. 105:1689-1697.

24. Issekutz, A.C. 1983. Removal of gram-negative endotoxin from solutions by affinity chromatography. J. Immunol. Methods. 61:275-281.

25. Miller, S. 1991. PhoP/PhoQ: macrophage-specific modulators of Salmonella virulence? Mol. Microbiol. 5:2073-2078.

26. Pegues, D., Hantman, M., Behleu, I., and Miller, S. 1995. PhoP/PhoQ transcriptional repression of $S$. typhimurium invasion genes: evidence for role in protein secretion. Mol. Microbiol. 17:169-181.

27. Kubori, T., Sukhan, A., Aizawa, S.I., and Galan, J.E. 2000. Molecular characterization and assembly of the needle complex of the Salmonella typhimurium type III protein secretion system. Proc. Natl. Acad. Sci. USA. 97:10225-10230.

28. Eckmann, L., Kagnoff, M., and Fierer, J. 1993. Epithelial cells secrete the chemokine interleukin-8 in response to bacterial entry. Infect. Immun. 61:4569-4574.

29. Eaves-Pyles, T., Szabo, C., and Salzman, A.L. 1999. Bacterial invasion is not required for activation of NF-kappaB in enterocytes. Infect. Immun. 67:800-804.

30. Calladine, C.R. 1975. Construction of bacterial flagella. Nature. 255:121-124.

31. Daig, R., et al. 1996. Increased interleukin 8 expression in the colon mucosa of patients with inflammatory bowel disease. Gut. 38:216-222.

32. Mazzucchelli, L., et al. 1994. Expression of interleukin-8 gene in inflammatory bowel disease is related to the histological grade of active inflammation. Am. J. Pathol. 144:997-1007.

33. Sansonetti, P.J., Arondel, J., Huerre, M., Harada, A., and Matsushima, K. 1999. Interleukin-8 controls bacterial transepithelial translocation at the cost of epithelial destruction in experimental shigellosis. Infect. Immun. 67:1471-1480.

34. Van Asten, F.J., Hendriks, H.G., Koninkx, J.F., Van der Zeijst, B.A., and Gaastra, W. 2000. Inactivation of the flagellin gene of Salmonella enterica serotype Enteritidis strongly reduces invasion into differentiated Caco2 cells. FEMS Microbiol. Lett. 185:175-179.

35. Ciacci-Woolwine, F., Blomfield, I.C., Richardson, S.H., and Mizel, S.B. 1998. Salmonella flagellin induces tumor necrosis factor alpha in a human promonocytic cell line. Infect. Immun. 66:1127-1134.

36. Fernandez, L.A., and Berenguer, J. 2000. Secretion and assembly of regular surface structures in Gram-negative bacteria. FEMS Microbiol. Rev. 24:21-44 